\title{
Liquid crystal dynamics in the isotropic phase
}

\author{
S. D. Gottke, David D. Brace, and Hu Cang \\ Department of Chemistry, Stanford University, Stanford, California 94305 \\ Biman Bagchi \\ Solid State and Structural Chemistry Unit, Indian Institute of Science, Bangalore 560 012, India \\ M. D. Fayer ${ }^{\mathrm{a})}$ \\ Department of Chemistry, Stanford University, Stanford, California 94305
}

\begin{abstract}
The dynamics in the isotropic phase of the liquid crystal 1-isothiocyanato-(4-propylcyclohexyl) benzene (3-CHBT) are investigated from very short time ( $\sim 1 \mathrm{ps})$ to very long time ( $>100 \mathrm{~ns})$ as function of temperature. The data decay exponentially only on the longest time scale ( $>10 \mathrm{~ns})$. The temperature dependence of the long time scale exponential decays is described well by the Landaude Gennes theory of the randomization of pseudo-nematic domains that exist in the isotropic phase of liquid crystals near the isotropic to nematic phase transition. Over the full range of times, the data are fit with a model function that contains a short time power law. The power law exponent is temperature independent over a wide range of temperatures. Integration of the function gives the empirical polarizability-polarizability (orientational) correlation function. A preliminary theoretical treatment of collective motions yields a correlation function that indicates that the data can decay as a power law at short times. The power law component of the decay reflects intradomain dynamics.
\end{abstract}

\section{INTRODUCTION}

Dynamics in liquid crystals in the isotropic phase have been studied widely in the last thirty years. Most of this work has focused on the slow time scale dynamics using measurements in the time and frequency domains. ${ }^{1-8}$ de Gennes, using Landau theory of phase transitions, explained the temperature dependence of the slowest time scale exponential orientational relaxation in relation to the nematic-isotropic (NI) transition. ${ }^{9}$ As the NI transition is approached from above, orientational relaxation slows dramatically. ${ }^{10} \mathrm{Al}-$ though the isotropic phase is macroscopically homogeneous, on a distance scale short compared to a correlation length, $\xi$, nematiclike order exists. Locally, molecules form "pseudonematic domains." Such domains persist in the isotropic phase up to $\sim 50 \mathrm{~K}$ over the NI transition temperature. The Landau-de Gennes (LdG) theory describes the orientational randomization of the domains. As the temperature is lowered, $\xi$ increases, and the orientational correlation function decays increasingly slowly, diverging at the NI transition. The LdG theory of long time scale orientational relaxation is well established experimentally using such techniques as depolarized light scattering, ${ }^{3}$ dynamic light scattering, ${ }^{4}$ magnetic, ${ }^{5}$ and electric birefringence, ${ }^{6}$ dielectric relaxation, ${ }^{7,8}$ and the optical Kerr effect. ${ }^{1,2,11}$

In contrast to the slow time scale dynamics, the nature of orientational relaxation on a time scale fast compared to the LdG relaxation time is not understood. On the fast time scale, the nematogens are locally ordered and are undergoing orientational relaxation in a nematically ordered environ-

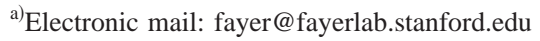

ment. Only a few experiments have focused on the faster time scale dynamics of liquid crystals. ${ }^{11-15}$ Compared to the experiments presented below, the earlier experiments had limited time range and signal-to-noise ratios $(\mathrm{S} / \mathrm{N})$. Nonetheless, the previous transient grating optical Kerr effect experiments, which measure the time derivative of the polarizability-polarizability correlation function, (except on $<1 \mathrm{ps}$ time scales, equivalent to the orientational correlation function) revealed power law decays at short times and the LdG exponential decay at long times. The data were fit with a model function consisting of a power law (short time intradomain dynamics) plus an exponential (domain randomization). Theoretical treatments based on single particle dynamics produced power law functions, ${ }^{16,17}$ but, as discussed below, do not describe the data appropriately.

The data presented below on the liquid crystal 1-isothiocyanato-(4-propylcyclohexyl)benzene (3-CHBT), taken with optical heterodyne detected optical Kerr effect experiments, provide a detailed view of the dynamics on all time scales. It is found that a more complicated model function than the one employed previously is required to fit the experimental data. As reported earlier, ${ }^{12,13,16}$ the short time behavior of the data is described by a power law, but the fitting function used here makes it possible to obtain a more accurate description of the power law and the data overall. Over a wide range of temperatures, the power law is essentially temperature independent. Integration of the model function provides an empirical orientational correlation function over the full range of times. This model correlation function does not contain a power law, but its derivative does. A preliminary theoretical analysis that calculates the orientational correlation function on fast time scales is based 
on a description of the collective orientational relaxation. The theoretical correlation function is not a power law, but its derivative displays a power law like decay at short time.

\section{EXPERIMENT}

Optical heterodyne-detected optical Kerr effect (OHDOKE) spectroscopy ${ }^{18}$ was used to measure the liquid crystal orientational relaxation. A pump pulse creates a timedependent optical anisotropy that is monitored via a heterodyne detected probe pulse with a variable time delay. The OHD-OKE experiment measures the system's impulse response function, which is the derivative of the polarizability-polarizability (orientational) correlation function. ${ }^{19-22}$ The methods for the analysis of OHD-OKE data have been described in detail. ${ }^{23}$ The Fourier transform of the OHD-OKE signal is directly related to data obtained from depolarized light scattering, ${ }^{24}$ but the time domain OHD-OKE experiment can provide better S/N over a broader range of times for experiments conducted on very fast to moderate time scales.

To observe the full range of liquid dynamics, at each temperature several sets of experiments were performed with different pulse lengths and delays. For times $t<30 \mathrm{~ns}$, a mode-locked $5 \mathrm{kHz}$ Ti:Sapphire laser/regenerative amplifier system was used ( $\lambda=800 \mathrm{~nm}$ for both pump and probe). The pulse length was adjusted from 75 to 1 ps to improve $\mathrm{S} / \mathrm{N}$. The shortest pulses were used for times 100 fs to a few tens of ps. For longer times, a few ps to $600 \mathrm{ps}$, the pulses were lengthened to $1 \mathrm{ps}$. The longer pulses produce more signal for the longer time portions of the data. For intermediate times, the pulse compression was bypassed, and a 100 ps pulse was used with a long delay line to obtain times from 100 ps to 30 ns. For even longer times, a CW diode laser was used for probing, and a fast digitizer ( 2 ns per point) recorded the data. The scans taken over various time ranges always overlapped substantially permitting the data sets to be merged by adjusting only their relative amplitudes. Great care was taken, and innumerable tests were made, to assure that the data sets could be combined without error or ambiguity. Additional experimental details have been published recently. ${ }^{25}$

3-CHBT was purchased from Aldrich and used without further purification except filtration through a $0.2 \mu \mathrm{m}$ disc filter to reduce light scattering. The sample was sealed under vacuum in a $1 \mathrm{~cm}$ glass cuvette. The cuvette was held in a constant flow cryostat where the temperature was controlled to $\pm 0.1 \mathrm{~K}$. Experiments were performed in the isotropic phase from 315 to $355 \mathrm{~K}$.

\section{RESULTS AND DISCUSSION}

Figure 1 is a data set taken at $317 \mathrm{~K}\left(T_{\mathrm{NI}}=314.7 \mathrm{~K}\right)$ shown on a $\log -\log$ scale. The data span the range of times from 1 ps to $200 \mathrm{~ns}$, that is, over five decades. As discussed further below, at short time the decay is a power law. It then becomes more gradual at intermediate times of several nanoseconds, almost appearing as if the decay is approaching a plateau on the log plot. At still longer times, the decay is an

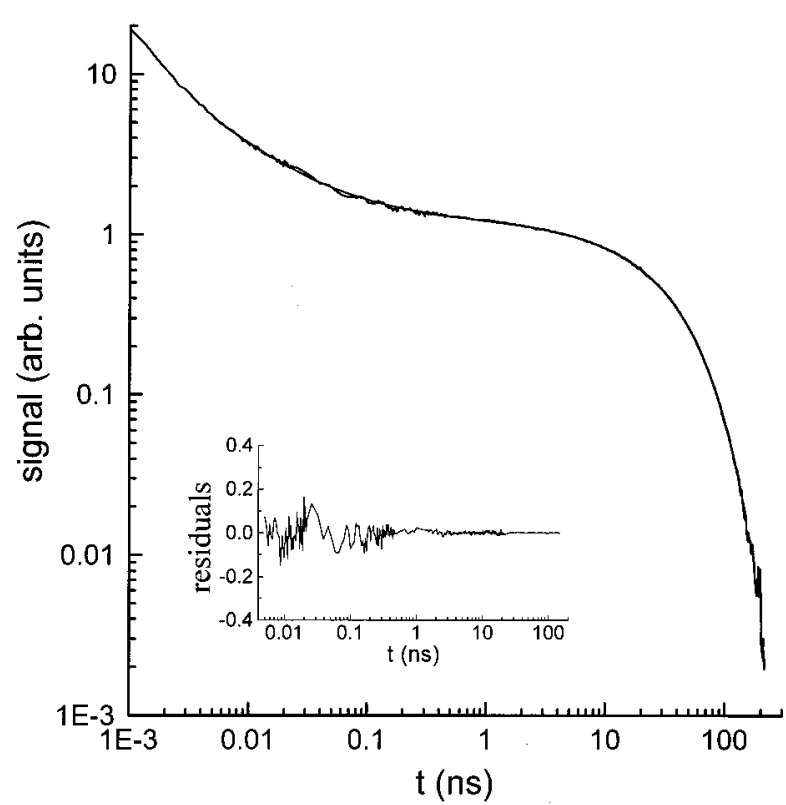

FIG. 1. Optical heterodyne detected optical Kerr effect data displaying the time dependence of orientational dynamics of the liquid crystal, 3-CHBT at $317 \mathrm{~K}$ on a $\log$ plot. The data span the time range $1 \mathrm{ps}$ to $200 \mathrm{~ns}$. At short time, the data decay as a power law. At long time, the data decay exponentially. Also shown is a fit to the data using Eq. (1). The fit is very good over the broad time range. The residuals of the fit are shown in the inset.

exponential. The long time portion of the data is a single exponential decay (see below) as expected from the LdG theory.

The data can be divided into two time regimes, that is, the LdG relaxation time scale and times fast compared to the LdG relaxation time, $\tau_{\mathrm{LdG}}$. The qualitative explanation for the two time regimes has been discussed previously. ${ }^{12}$ The following is a brief explanation. In the isotropic phase, the macroscopic liquid has an order parameter, $S=0$, that is, macroscopically there is no orientational order. However, on a distance scale short compared to $\xi$, and on a time scale short compared to $\tau_{\mathrm{LdG}}$, there is pseudo-nematic order, which can be characterized by a local order parameter, $S_{L}$, relative to a local director. When the pump pulse $E$-field is applied, the nematogens experience a torque that produces a slight alignment with the field. When the field is removed, the macroscopic system is left with $S \neq 0$. Field free evolution will reestablish $S=0$.

To understand the origin of the dynamical time scales, it is necessary to consider the problem microscopically. There are two contributions to the relaxation, intradomain relaxation and domain randomization (LdG relaxation). The $E$-field induces a small net alignment of the individual molecules. The molecular alignments change the local order parameter, $S_{L}$. Unlike $S$, which is a macroscopic parameter, $S_{L}$, is nonzero prior to the application of the optical field. $S_{L}$ defines the local nematic structure relative to the local director assigned with a given pseudo-nematic domain. Initially, $S_{L}=S_{L}^{0}$. Immediately following the application of the field, $S_{L}>S_{L}^{0}$. ( $S_{L}$ can also be $<S_{L}^{0}$, depending on the direction of the local director relative to the field direction.) The small alignment of the molecules with the field changes $S_{L}$, and it 
also rotates the direction of the local director. Fast intradomain relaxation occurs, restoring the local order parameter to $S_{L}^{0}$. Relaxation of the perturbed local order back to $S_{L}^{0}$ occurs on the fast time scales but leaves the ensemble of domain local directors still slightly aligned with the direction defined by the $E$-field. This long-lived anisotropy can only decay by randomization of the domains and is responsible for the long time decay described by LdG theory.

Also shown in Fig. 1 is a fit to the data using the model function

$$
f(t)=e^{-t / \tau}\left[a+e^{-t / \gamma}\left(b+\left(\frac{t}{t_{\delta}}\right)^{-p}\right)\right] .
$$

The exponential multiplying the terms in square brackets is the long time decay, with $\tau=\tau_{\mathrm{LdG}}$ in the temperature range near the NI transition where LdG theory is applicable. The power law term, with $t$ scaled by $t_{\delta}$, which sets the magnitude of the power law portion, describes the short time behavior (longer than $\sim 1 \mathrm{ps}$ ). The additional exponential, with the decay constant $\gamma$, is necessary to obtain the flattening of the curve prior to the LdG decay. All of the terms inside the square brackets describe the intradomain, non-LdG, decay. The intradomain decay leaves a residual anisotropy of amplitude $a$, which then decays via the domain randomization with $\tau_{\mathrm{LdG}}$. The fit to the $317 \mathrm{~K}$ data shown in Fig. 1 uses the parameters: $\tau_{\mathrm{LdG}}=36.8 \mathrm{~ns}, \gamma=9.55 \mathrm{~ns}, p=0.73, a=1.006$, $b=0.175, t_{\delta}=0.035 \mathrm{~ns}$. Note that the function in Eq. (1) does an excellent job of describing the data over a wide range of times. The inset shows the residuals of the fit to the data. The function given in Eq. (1) works at all temperatures, and, as will be shown subsequently ${ }^{26}$ it describes the data equally well for three different liquid crystals. The previous function used to describe the data, the simple sum of a power law and an exponential, does not accurately reproduce the intermediate time scale data (just prior to the LdG decay) nor does it properly describe the very long time behavior. It also contains a fundamental conceptual flaw. At sufficiently high temperature, the LdG decay becomes very fast. At even higher temperature, domains cease to exist, and the decay should become a single fast exponential. With a functional form that is the sum of a power law and an exponential (the function used in prior work ${ }^{12,13,16}$ ), making the exponential very fast leaves a long time decay that is a power law. This is contrary to observation. Even for the decays at moderate temperature, at sufficiently long time, the simple sum of a power law and an exponential leaves a long power law tail. The present data are good enough and go out far enough that comparison to the simple sum deviates from the data at long time. The function in Eq. (1) does not have these problems. The intradomain terms decay, leaving a residual anisotropy that decays exponentially. If the LdG decay becomes very fast, then the entire function decays exponentially.

Figure 2 displays the temperature dependent data on a $\log$ plot. The data sets have been offset vertically for clarity. The lowest temperature is the top curve, and each curve moving downward is at successively higher temperature. Note that the shortest time portions of each curve look identical. At long time, as the temperature is increased, the knee

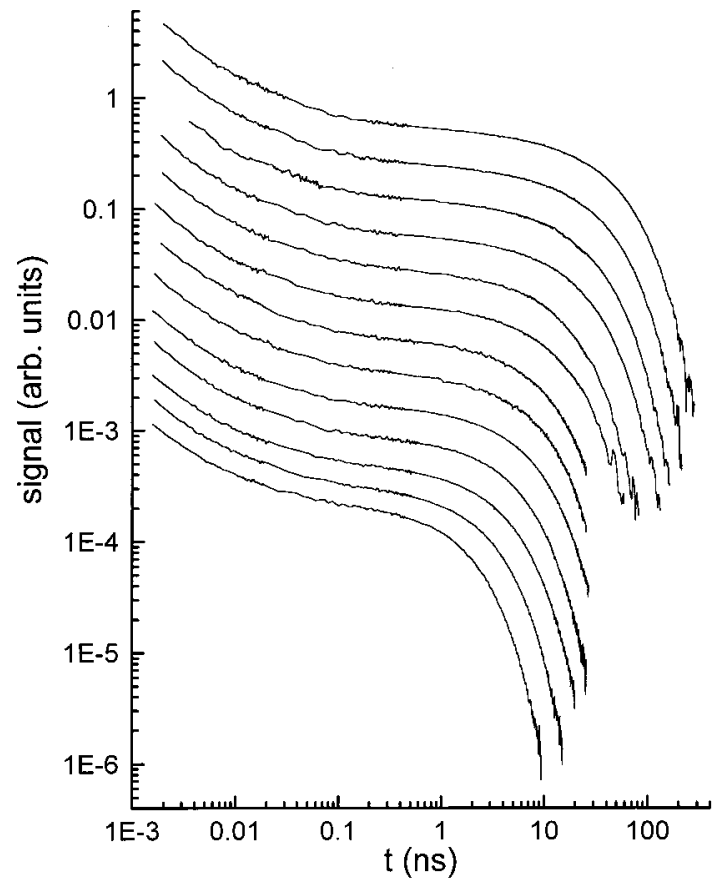

FIG. 2. Temperature dependent data sets displayed on a log plot. The data sets have been offset for clarity of presentation. Starting with the top most curve, the temperatures for the data sets are 316.0, 317.0, 318.0, 319.1, $321.5,323.0,325.1,327.0,329.0,333.1,337.0,344.0$, and $354.0 \mathrm{~K}$.

in the data (onset of the exponential decay) moves to shorter time. The shortest time portion of the data is a power law. Between the short power law and the long time exponential, in every data set is a region that appears to decay very gradually on the log plot.

Figure 3 displays the long time exponential decay time, $\tau_{\mathrm{LdG}}$, as a function of temperature. The inset displays the long time portion of the data at $317 \mathrm{~K}$ on a semilog plot. A line is shown passing through the data. As can be seen in the inset, the long time portion of the data is described very well as an exponential decay. Using Eq. (1) to fit the data or fitting only the long time tail to an exponential gives identical results for $\tau_{\mathrm{LdG}}$. The LdG theory predicts ${ }^{10}$

$$
\tau_{\mathrm{LdG}} \propto \frac{V_{\mathrm{eff}}^{*} \eta(T)}{k_{B}\left(T-T^{*}\right)^{\gamma}},
$$

where $\eta(T)$ is the viscosity (see Table I for viscosity data), $T$ is the temperature, $T^{*}$ is the transition temperature, which is generally a degree or more below $T_{\mathrm{NI}},{ }^{10} k_{B}$ is the Boltzmann constant, $V_{\text {eff }}^{*}$ is the effective volume., ${ }^{3,27}$ The exponent $\gamma$ has been shown to be $1,{ }^{11}$ indicating the validity of mean-field theory. ${ }^{10}$ The curve through the data in Fig. 3 is obtained from Eq. (2) using $V_{\text {eff }}^{*}$ as a scaling parameter. $V_{\text {eff }}^{*}$ does not change the shape of the curve; it only sets the magnitude. $T^{*}$ was found to be $312.5 \mathrm{~K}$. Clearly, the long time behavior is described very well by LdG theory, as is expected.

To reveal the power law component of the data more clearly, using the fit with Eq. (1), the contributions from the two exponentials can be removed. Figure 4 displays the power law portion of one data set (the data in Fig. 1). In the figure, the power law is shown from 3 ps to $2 \mathrm{~ns}$. The power 


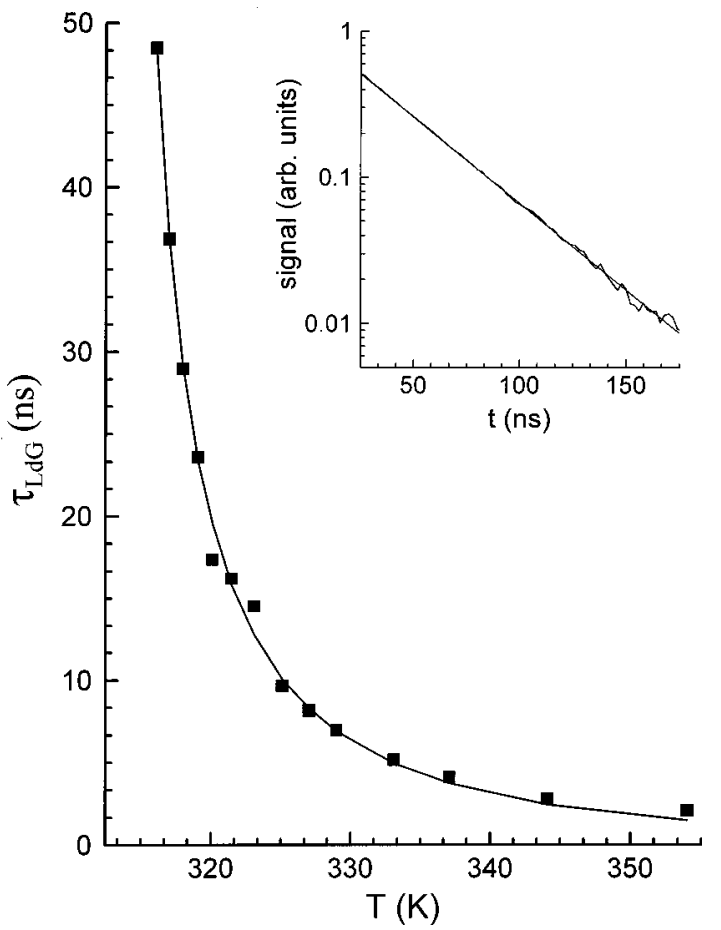

FIG. 3. The long time scale exponential decay time as a function of temperature. The temperature dependence of the decay time is predicted by the Landau-de Gennes theory. The solid curve through the data is the theoretically predicted LdG curve. The inset shows the long time portion of the 317 $\mathrm{K}$ data set on a semilog plot with a line through the data showing the decay is exponential.

law exponent obtained from the fit and displayed in the plot is 0.73 . Figure 5(a) displays the power law exponent as a function of temperature for the range of temperatures studied. Within experimental error, the power law is temperature independent. The value of the exponent is $0.75 \pm 0.03$. In previous studies of liquid crystals, ${ }^{12,13}$ the power law was also found to be temperature independent, but only at temperatures sufficiently close to the NI transition temperature. In the current study, and in studies of other liquid crystals that will be reported subsequently, ${ }^{26}$ the power law is found

TABLE I. Temperature dependent viscosity.

\begin{tabular}{cc}
\hline \hline Temperature $(\mathrm{K})$ & Viscosity $(\mathrm{cP})$ \\
\hline 318.15 & 9.67 \\
320.25 & 9.21 \\
322.65 & 8.53 \\
325.65 & 7.79 \\
329.15 & 7.12 \\
333.65 & 6.49 \\
338.15 & 5.75 \\
341.65 & 5.24 \\
344.65 & 4.93 \\
348.65 & 4.53 \\
351.65 & 4.23 \\
355.15 & 4.02 \\
358.15 & 3.82 \\
361.65 & 3.52 \\
364.65 & 3.41 \\
367.35 & 3.11 \\
370.65 & 3.01 \\
\hline \hline
\end{tabular}

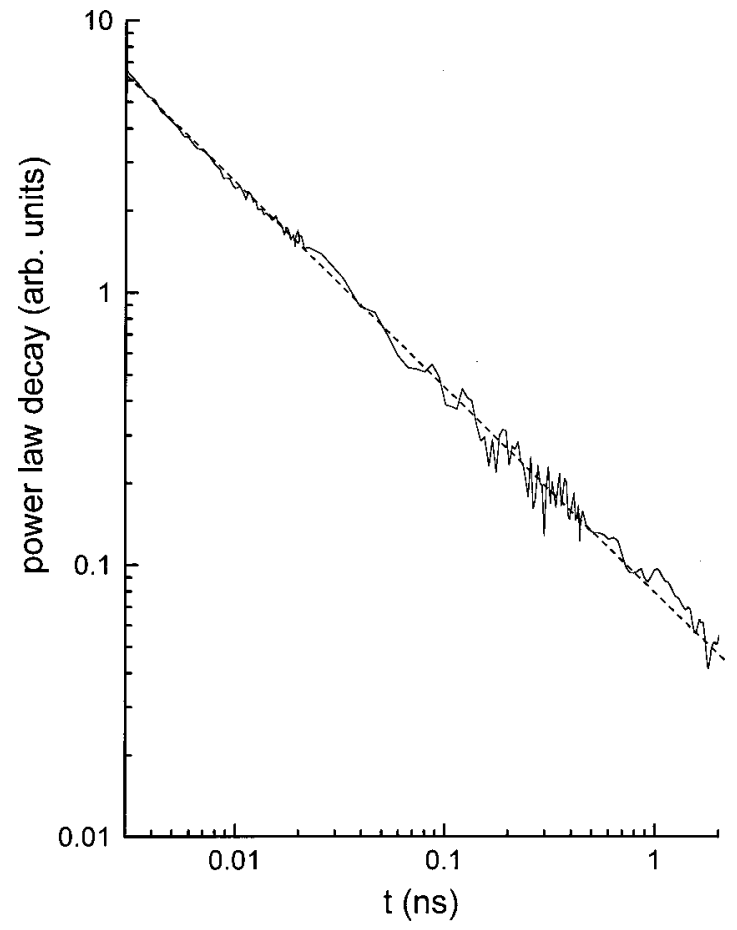

FIG. 4. The short time portion of the $317 \mathrm{~K}$ data with the exponential contributions removed [see Eq. (1)] on a log plot. The dashed line through the data shows that the decay is a power law from 3 ps to $2 \mathrm{~ns}$.

to be essentially temperature independent to temperatures further above the NI transition temperature. The previous work did not account for the intermediate time portion of the curve, which may have a weak temperature dependence. The
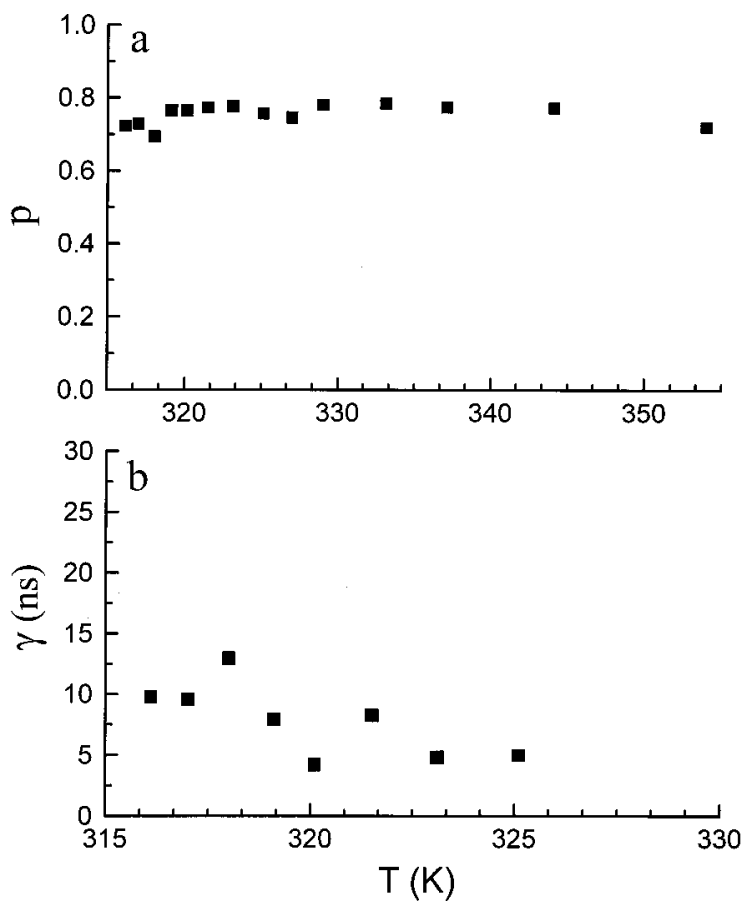

FIG. 5. (a) The power law exponent, $p$, obtained from fits of the data to Eq. (1), as a function of temperature. Within experimental error, the power law exponent is temperature independent, $p=0.75 \pm 0.03$. (b) The intermediate exponential decay constant $\gamma$ as a function of temperature. Within experimental error, $\gamma$ is either weakly temperature dependent or temperature independent. 
decay constant, $\gamma$, for the intermediate time scale exponential is shown in Fig. 5(b). While $\gamma$ is either temperature independent or very mildly temperature dependent, $\tau_{\mathrm{LdG}}$ is highly temperature dependent. For temperatures $>325 \mathrm{~K}$, the two decay constants could no longer be distinguished because $\tau_{\mathrm{LdG}}<\gamma$ and $a>b$. In Eq. (1), multiplying through by the outer exponential, $\exp (-t / \tau)$, gives three exponentials, two of which have a decay constant

$$
K=\frac{1}{\tau}+\frac{1}{\gamma}
$$

At the higher temperatures, $K=1 / \tau$.

One other study of 3-CHBT performed recently measured dielectric relaxation in the nematic and isotropic phases. ${ }^{28}$ Although the time resolution of these experiments is limited, and the temperature range in the isotropic phase extended to only $7 \mathrm{~K}$ above the nematic-isotropic phase transition, nevertheless, the data appear consistent with the results found here. Two relaxation times were measured to be faster than the Landau-de Gennes decay time. These could correspond to the intermediate and fast processes observed in the experiments presented here. Another study on a mixture of liquid crystals ${ }^{15}$ also displayed two decay processes in addition to the Landau-de Gennes decay.

The model function for the decay given in Eq. (1) describes the data well. This function is related to the time derivative of the polarizability-polarizability correlation function. By integrating Eq. (1) and normalizing the function at $t=0$, an empirical correlation function is obtained, that is

$C(t)=\frac{a \tau e^{-t / \tau}+(b / K) e^{-K t}+\left(1 / t_{\delta}\right)^{-p} K^{p-1} \Gamma(1-p, K t)}{a \tau+(b / K)+\left(1 / t_{\delta}\right)^{-p} K^{p-1} \Gamma(1-p)}$,

where $K$ is defined above, and $\Gamma(x)$ is the gamma function, and $\Gamma(x, y)$ is one of the definitions of the incomplete gamma function ${ }^{29}$

$$
\Gamma(x, y)=\int_{y}^{\infty} e^{-s} s^{x-1} d s .
$$

All of the parameters in the expression for the power law are obtained from the fits to the experimental data. While the correlation function given in Eq. (3) is empirical, a correlation function derived theoretically from first principles will display the same time dependent behavior. Therefore, Eq. (3) is useful for comparison to theoretical calculations of the full correlation function.

\section{PRELIMINARY THEORY OF SHORT TIME $\left(\ll \tau_{\text {LdG }}\right)$ DECAY}

Previous theoretical examination of the short time behavior treated the problem calculating a single particle correlation function. ${ }^{16,17}$ These calculations did yield power laws. However, the correlation functions were compared directly to the data; the required derivative was not taken. Once the derivative is taken, the calculated decays are much steeper than the data, that is, the power law exponent in the derivative function is too large. More important, the single particle correlation function should only apply at extremely short time. The time scales observed in the experiments reflect the collective dynamics of the local nematically ordered structures. Here we present a preliminary theory of the collective correlation function at short times, the derivative of which decays approximately as a power law on the fast time scale.

As the function measured in optical Kerr experiments is the time derivative of the total polarizability-polarizability time correlation function, the response is determined primarily by the time correlation function of the second rank spherical harmonics, $Y_{2 m}\left(\Omega_{i}(t)\right)$, where $\Omega_{i}(t)$ is the orientation of $i$ th nematogen at time $t .^{30}$ Therefore, we define a collective function by ${ }^{31,32}$

$$
Y_{2 m}(k, t)=\sum e^{i k r_{i}(t)} Y_{2 m}\left(\Omega_{i}(t)\right),
$$

where the sum is over all molecules in the system. We further define the orientational time correlation function $C_{2 m}(k, t)$ by the following relation: ${ }^{31,32}$

$$
C_{2 m}(k, t)=\left\langle Y_{2 m}(-k, 0) Y_{2 m}(k, t)\right\rangle,
$$

where the angular brackets denote an ensemble average. In Kerr experiments, we measure the collective, that is, the $k$ $=0$ limit of $C_{2 m}(k, t)$. In the present work, we shall consider only the $C_{20}(k, t)$ correlation function. It is convenient to work in the Laplace frequency plane where $C_{20}(k, z)$ is defined by

$$
C_{20}(k, z)=\int_{0}^{\infty} d t e^{-z t} C_{20}(k, t) .
$$

One can derive the following expression for $C_{20}(k, z)$ from a general molecular hydrodynamic approach: ${ }^{32-34}$

$$
C_{20}(k, z)=\frac{C_{20}(k)}{z+\Sigma_{20}(k, z)},
$$

where the generalized diffusion coefficient, $\Sigma_{20}(k, z)$, is given by ${ }^{34}$

$$
\sum_{20}(k, z)=\frac{6 k_{B} T f_{220}(k)}{\left[z+\Gamma_{R}(k, z)\right] I}+\frac{k_{B} T k^{2} f_{220}(k)}{\left[z+\Gamma_{T}(k, z)\right] m},
$$

where $k_{B} T$ is Boltzmann constant times temperature, $I$ is the moment of inertia, and $m$ is the mass. $\Gamma_{R}(k, z)$ and $\Gamma_{T}(k, z)$ are the wave vector and frequency dependent rotational and translational memory kernels, respectively. $f_{220}(k)$ is the dimensionless orientational caging parameter defined by

$$
f_{220}(k)=1-(\rho / 4 \tau) c_{220}(k) \equiv 1 / S_{220}(k),
$$

where $c_{220}(k)$ is the $(220)$ component of wave vector and orientational dependent direct correlation function and $\rho$ is the number density of nematogens. Note that as the isotropic-nematic phase transition is approached from the isotropic side, $f_{220}(k)$ undergoes a pronounced softening. The divergence of $S_{220}(k=0)$ is preceded by the weakly first order isotropic to nematic phase transition. ${ }^{35-38}$ However the growth of $S_{220}(k=0)$ as the NI transition is approached (from the isotropic side) leads to the softening of $f_{220}(k$ $=0$ ). This softening is the primary reason for the stretching of the relaxation in the LdG theory, which is recovered from 
the present theory at long time when $\Sigma_{20}(k, z)$ is replaced by $\Sigma_{20}(k=0, z=0)$, and $\Gamma_{R}$ by its single particle limit, that is rotational friction, $\xi_{R}$. Under the above limiting conditions, the orientational correlation function decays as a single exponential with a rate equal to $6 D_{R} / S_{220}(k=0)$.

The situation is different at times short compared to $\tau_{\mathrm{LdG}}$, where $\Sigma_{20}(k, z)$ undergoes sharp variation with the Laplace frequency $z$, in particular near the NI transition when it is approached from the isotropic phase. This behavior of $\Sigma_{20}(k, z)$ can be captured by mode coupling theory ${ }^{39}$ because this variation in $\Sigma_{20}(k, z)$ is due to the coupling of the rotational velocity field (vortices) to the orientational density fluctuation. To this end, we split the rotational friction $\Gamma_{R}$ into a short time part, $\Gamma_{0}$, and a singular part, $\Gamma^{\text {sing }}$

$$
\Gamma_{R}=\Gamma_{0}+\Gamma^{\text {sing }},
$$

where $\Gamma_{0}$ is determined by local, short-range interactions. ${ }^{40}$ $\Gamma^{\text {sing }}$ is the part which is coupled to show collective orientational fluctuations, that is, to the fluctuations of the order parameter. $\Gamma^{\text {sing }}$ can be calculated using Kirkwood's formula, ${ }^{32,33}$

$$
\Gamma^{\text {sing }}=\frac{1}{2 I k_{B} T} \int_{0}^{\infty} d t \frac{1}{4 \pi V} \int d r d \Omega\langle N(r, \Omega, 0) N(r, \Omega, t)\rangle .
$$

When the torque, $N$, is obtained from density functional theory, ${ }^{32,34}$ we get the following expression for $\Gamma^{\text {sing. }}$

$$
\Gamma^{\text {sing }}=\frac{3 k_{B} T \rho}{8 \pi^{3} I} \int_{0}^{\infty} d k k^{2} c_{220}^{2}(k) C_{20}(k, t) .
$$

This is a typical MCT expression. ${ }^{40}$ Note that $C_{20}(k, t)$ in turn depends on $\Gamma^{\text {sing }}$. Thus, a self-consistent iterative calculation is called for. Note also that $\Gamma^{\text {sing }}$ derives contributions from all length scales. Near the NI transition, however, $\Gamma^{\text {sing }}$ is expected to be dominated by the long wavelength (i.e., small $k$ value) modes. We next approximate $C_{20}(k, t)$ by

$$
C_{20}(k, t)=S_{220}(k) \exp \left(-6 D_{R} t / S_{220}(k)\right),
$$

where $D_{R}$ is the rotational diffusion coefficient of the nematogens.

Near the NI transition

$$
c_{220}(k=0) \approx(4 \pi / \rho)^{2},
$$

and $S_{220}(k)$ grows at small $k$ as ${ }^{36-38}$

$$
S_{220}(k \rightarrow 0) \approx 1 / B^{2} k^{2},
$$

where $B$ is related to the second derivative of the direct correlation function $c_{220}(k)$ at $k=0$, that is

$$
B^{2}=\frac{\rho}{4 \pi}\left(\frac{d^{2} c_{220}}{d k^{2}}\right)_{k=0} .
$$

The mode coupling contribution can now be obtained by performing the $k$-integration, which gives the following expression:

$$
\Gamma^{\operatorname{sing}}(t) \approx \frac{A_{0}}{\sqrt{t}},
$$

with

$$
A_{0}=\frac{3 k_{B} T}{\pi I \rho B^{3}}\left(\pi / 6 D_{R}\right)^{1 / 2} .
$$

Laplace transformation of $\Gamma^{\operatorname{sing}}(t)$ can be easily performed to obtain

$$
\Gamma^{\operatorname{sing}}(z)=\frac{A}{\sqrt{z}},
$$

where $A=A_{0} \pi^{1 / 2}$. At small $z, \Gamma^{\text {sing }}$ dominates, and we have

$$
\sum_{20}(k=0, z) \approx \frac{k_{B} T f_{220}(k=0)}{I A} z^{1 / 2} .
$$

The above expression for $\Sigma_{20}$ leads to the following expression for $C_{20}(z)$ at small $z$

$$
C_{20}(z)=\frac{1}{z+a z^{1 / 2}},
$$

where

$$
a=k_{B} T f_{220}(k=0) / I A .
$$

Substituting the definition of $A$ gives

$$
a=\rho B^{3}\left(6 D_{R}\right)^{1 / 2} f_{220}(k=0) / 3 .
$$

The above expression for $C_{20}(z)$ can be easily Laplace inverted to obtain

$$
C_{20}(t)=\exp \left(a^{2} t\right) \operatorname{erfc}\left(a t^{1 / 2}\right),
$$

where erfc is complementary error function. ${ }^{29}$ Equation (22) for $C_{20}(t)$ is the main result of the theoretical analysis. It is expected to be valid in a time window that is short compared to $\tau_{\mathrm{LdG}}$ but long compared to the ultrashort time scale of collisional dynamics. Equation (22) shows that at relatively short times, the polarizability-polarizability time correlation function will have a weak time dependence (see below).

The present analysis suggests that the weak time dependence on time scales short compared to $\tau_{\mathrm{LdG}}$ is a direct consequence of pseudo-nematic time domain formation as the NI transition is approached from the isotropic phase. The pseudo-nematic domains give rise to slow, local orientational density fluctuations, which in turn affect the orientational friction because the torque-torque correlation function is coupled to these fluctuations. The influence of the domains on the local orientational density fluctuations is reflected in a strong frequency dependence of the rotational friction in the low-frequency range. The strong frequency dependence of friction acts in the opposite manner to the usual decay from the short-range interactions-nearly canceling it. At long times, $\left(t \geqslant \tau_{\mathrm{LdG}}\right)$ this frequency dependence dies down, and the LdG theory should be recovered. One, therefore, expects a nonexponential crossover region where mild decay at short times will go over to the long time scale exponential decay. This crossover region is akin to the von Schweidler region $^{41,42}$ of the dynamics observed in supercooled liquids. ${ }^{25,43}$ It arises from essentially the same reason, that is, the stretching of relaxation at intermediate times. 


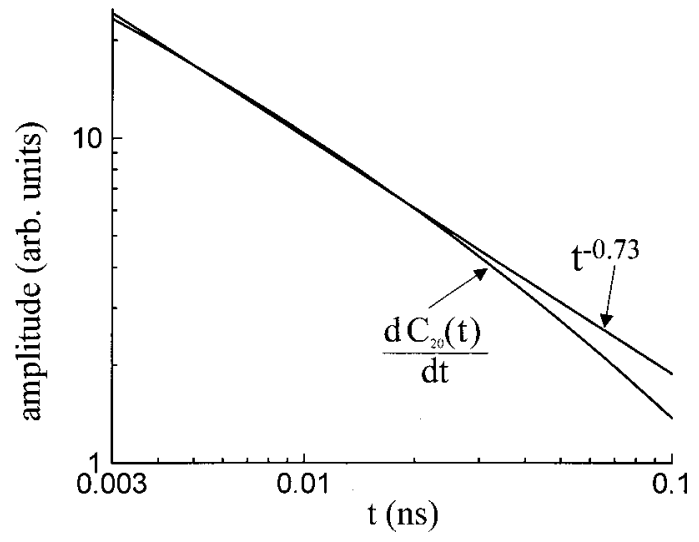

FIG. 6. The time derivative of the theoretical correlation function, $C_{20}(t)$ [Eq. (22)]. Also shown is a $t^{-0.73}$ power law. At short time, the derivative of the correlation function decays essentially as a power law. By the proper choice of the parameter, $a$, the decay can be made to match the short time portion of the experimental data.

The correlation function given in Eq. (22) has a very different form from the experimentally derived empirical correlation function given in Eq. (3). The function given in Eq. (3) spans the full time range from $\mathrm{ps}$ to $t>\tau_{\mathrm{LdG}}$. Its derivative, Eq. (1), describes the data well (see Fig. 1). Figure 6 shows the derivative of the theoretical correlation function [Eq. (22)] with $a=3$. The experimental data begins with a power law with exponent of $\sim-0.73$ (see Fig. 4). Figure 6 also displays a power law $t^{-0.73}$. The derivative of the correlation function is almost a power law at short time, from 3 to $\sim 30 \mathrm{ps}$. After that, it begins to tail off. The value of the parameter, $a$, was selected to produce a curve which is similar to the power law portion of the data at short time. Figure 6 is not intended to be a fit to the data. It is only used to demonstrate that the correlation function given in Eq. (22) can have the appropriate short time behavior.

\section{CONCLUDING REMARKS}

In this paper, we have presented experiments and theory that investigate the orientational dynamics of liquid crystals in the isotropic phase near the nematic-isotropic phase transition temperature. The liquid crystal 3-CHBT was studied using optical heterodyne detected optical Kerr effect experiments, which provided very high quality data over a very broad range of times, that is, from 1 ps to 200 ns. The experiment measures the time dependence of the derivative of the polarizability-polarizability (orientational) correlation function. At short times, the data decay as a power law, $t^{-p}$, with a temperature independent exponent of $p=0.75 \pm 0.03$. At long time, the data decay exponentially as described by Landau-de Gennes theory. The short time scale dynamics reflect the collective orientational relaxation of the nematogens in an environment that is locally nematic. The long time dynamics reflect the randomization of the pseudo-nematic domains. While the long time dynamics are highly temperature dependent, with the decay time growing rapidly as the temperature is lowered and the isotropic-nematic phase transition is approached from the isotropic side, the power law is temperature independent within experimental error. The lack of temperature dependence suggests that the local pseudonematic structure is virtually temperature independent. The size of the domains grow as the temperature is decreased toward the phase transition, reflected by an increasing correlation length, but the data demonstrates that the dynamics at relatively short time are temperature independent or at most, very weakly temperature dependent.

The data were fit to a model fitting function that was able to reproduce the data extremely well (see Fig. 1) over the full range of times and temperatures. Integration of the fitting function yields an empirical function for the polarizability-polarizability correlation function. A true first principles theoretical correlation function will have the same shape as the empirical correlation function. A preliminary first principles theory of the correlation function was presented. This theory describes the collective orientational dynamics of the nematogens on a relatively short time scale only (i.e., $t<t_{\mathrm{LdG}}$ ). Previous theoretical treatments examined the single particle correlation function, which may only be appropriate on extremely short time scales. The collective correlation function [Eq. (22)] has a very weak time dependence at short times. The derivation of the correlation function decays essentially as a power law at short time (Fig. 6), and by the proper choice of the parameter $a$ [Eq. (21)], it can be brought into accord with the short time portion of the data. Further detailed theoretical studies will more fully investigate the correlation function on all time scales and its temperature dependence. Additional experiments will be presented that show that the dynamical behavior observed for 3 -CHBT is very similar to that of other liquid crystals. ${ }^{26}$

\section{ACKNOWLEDGMENTS}

One of the authors (B.B.) would like to thank the Theoretical Chemistry Institute of the University of Texas at Austin for support of his stay in the United States, which contributed to this work. This research was supported by the National Science Foundation (DMR-0088942).

${ }^{1}$ G. K. L. Wong and Y. R. Shen, Phys. Rev. Lett. 30, 895 (1973).

${ }^{2}$ E. G. Hanson, Y. R. Shen, and G. K. L. Wong, Phys. Rev. A 14, 1281 (1976)

${ }^{3}$ T. D. Gierke and W. H. Flygare, J. Chem. Phys. 61, 22331 (1974).

${ }^{4}$ T. W. Stinson III and J. D. Litster, Phys. Rev. Lett. 25, 503 (1970).

${ }^{5}$ J. D. Litster and T. W. Stinson III, J. Appl. Phys. 41, 996 (1970).

${ }^{6}$ J. C. Fillippini and Y. Poggi, Phys. Lett. A 65, 30 (1978).

${ }^{7}$ W. H. de Jeu, in Solid State Physics, edited by L. Liebert (Academic, New York, 1978), p. 109.

${ }^{8}$ H. Kresse, in Advances in Liquid Crystals, edited by G. H. Brown (Academic, New York, 1983), Vol. 6, p. 109.

${ }^{9}$ P. G. de Gennes, Phys. Lett. A 30, 454 (1969).

${ }^{10}$ P. G. de Gennes, The Physics of Liquid Crystals (Clarendon, Oxford, 1974).

${ }^{11}$ J. J. Stankus, R. Torre, C. D. Marshall, S. R. Greenfield, A. Sengupta, A. Tokmakoff, and M. D. Fayer, Chem. Phys. Lett. 194, 213 (1992).

${ }^{12}$ J. J. Stankus, R. Torre, and M. D. Fayer, J. Phys. Chem. 97, 9478 (1993).

${ }^{13}$ F. W. Deeg, S. R. Greenfield, J. J. Stankus, V. J. Newell, and M. D. Fayer, J. Chem. Phys. 93, 3503 (1990).

${ }^{14}$ R. Torre and S. Californo, J. Chim. Phys. 93, 1843 (1996).

${ }^{15}$ R. Torre, F. Tempestini, P. Bartolini, and R. Righini, Philos. Mag. B 77(2), 645 (1998).

${ }^{16}$ A. Sengupta and M. D. Fayer, J. Chem. Phys. 102, 4193 (1995).

${ }^{17}$ S. Ravichandran, A. Perera, M. Moreau, and B. Bagchi, J. Chem. Phys. 109(17), 7349 (1998). 
${ }^{18}$ D. McMorrow, W. T. Lotshaw, and G. A. Kenney-Wallace, IEEE J. Quant. Elec. 24(2), 443 (1988).

${ }^{19}$ Y. X. Yan, L. G. Cheng, and K. A. Nelson, Adv. Infrared and Raman Spectrosc. 16, 299 (1987)

${ }^{20}$ Y. X. Yan and K. A. Nelson, J. Chem. Phys. 87, 6240 (1987).

${ }^{21}$ Y. X. Yan and K. A. Nelson, J. Chem. Phys. 87, 6257 (1987).

${ }^{22}$ F. W. Deeg, J. J. Stankus, S. R. Greenfield, V. J. Newell, and M. D. Fayer, J. Chem. Phys. 90, 6893 (1989).

${ }^{23}$ D. McMorrow and W. T. Lotshaw, J. Phys. Chem. 95, 10395 (1991).

${ }^{24}$ Y. Kai, S. Kinoshita, M. Yamaguchi, and T. Yagi, J. Mol. Liq. 65-6, 413 (1995).

${ }^{25}$ S. D. Gottke, D. D. Brace, G. Hinze, and M. D. Fayer, J. Phys. Chem. B 105, 238 (2001).

${ }^{26}$ S. D. Fottke, H. Cang, B. Bagchi, and M. D. Fayer, J. Chem. Phys. (in preparation).

${ }^{27}$ G. R. Alms, T. D. Gierke, and W. H. Flygare, J. Chem. Phys. 61, 4083 (1974).

${ }^{28}$ J. Jadzyn, L. Hellemans, M. Stockhausen, C. Legrand, and G. Czechowski, Z. Naturforsch., A: Phys. Sci. 49, 1077 (1994).
${ }^{29}$ M. Abramowitz and I. A. Stegun, Handbook of Mathematical Functions (Dover, New York, 1970).

${ }^{30}$ G. R. Fleming, Chemical Applications of Ultrafast Spectroscopy (Oxford, New York, 1986).

${ }^{31}$ B. J. Berne, J. Chem. Phys. 62, 1154 (1975).

${ }^{32}$ B. Bagchi and A. Chandra, Adv. Chem. Phys. 80, 1 (1991).

${ }^{33}$ P. Boon and S. Yip, Molecular Hydrodynamics (McGraw-Hill, New York, 1980).

${ }^{34}$ B. Bagchi and R. Biswas, Adv. Chem. Phys. 109, 207 (1999).

${ }^{35}$ R. Zwanzig, J. Chem. Phys. 39, 1714 (1963).

${ }^{36}$ A. Perera, P. G. Kusalik, and G. N. Patey, J. Chem. Phys. 87, 1295 (1987).

${ }^{37}$ A. Perera, P. G. Kusalik, and G. N. Patey, Mol. Phys. 60, 77 (1987).

${ }^{38}$ A. Perera, G. Patey, and J. J. Weis, J. Chem. Phys. 89, 6941 (1988).

${ }^{39} \mathrm{U}$. Baluchani and M. Zoppi, Dynamics of the Liquid State (Clarendon, Oxford, 1994).

${ }^{40}$ B. Bagchi and S. Bhattacharyya, Adv. Chem. Phys. 116, 67 (2001).

${ }^{41}$ W. Götze and L. Sjögren, Rep. Prog. Phys. 55, 241 (1992).

${ }^{42}$ W. Götze, J. Phys.: Condens. Matter 11(10a), A1 (1999).

${ }^{43}$ G. Hinze, D. D. Brace, S. D. Gottke, and M. D. Fayer, J. Chem. Phys. 113(9), 3723 (2000). 